CaA NOTE MAT. No. 15
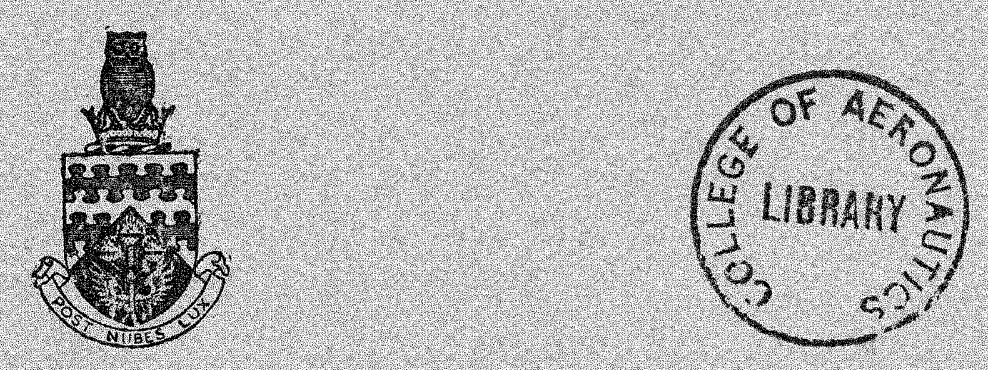

THE COLLEGE OF AERONAUTICS

C R A N F I EL D

SOME ASPECTS OF ANISOTROPIC PLASTICITY IN SHEET METALS by

R. Pearce 


\section{THE COILEGE OF AERONAUTICS}

\section{DEPARTMENT OF MATERIAIS}

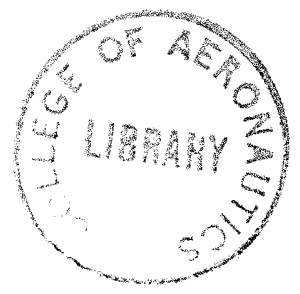

Some aspects of anisotropic plasticity in sheet metals

$$
\text { - by - }
$$

Roger Pearce, B.A., B.Sc., F.I.M.

\section{$S U M M A R Y$}

The prediction of the yielding and flow behaviour of materials under complex stress systems from tensile test or other easily determined data has been the aim of engineers for many years. The yield criteria of Tresca and then Mises for isotropic metals are useful, but the realisation that anisotropy is the rule rather than the exception, especially in sheet metals led to the examination of Hill's anisotropic theory by varlous workers. In the present paper the stress-strain curves of various sheet metals are determined in uniaxial and balanced biaxial tension. As far as yielding behaviour is concerned it is concluded that the theory is reasonably satism factory for materials where anisotropy is described with $r>1$, with certain anomalies for materials with $x<1$. As far as flow behaviour is concerned, the theory only applies for materials for $r>1$. Crossing of the uniaxial and biaxial curves is observed for certain metals at low strains and this is not predicted by the theory. More work is necessary on low-r materials to resolve these matters. 
Contents

Page No.

Summary

Introduction

1

Experimental

3

Results

4

Conclusions

4

Acknowledgements

5

Table

6

References

Figures 
Introduction

The mechanical properties of sheet metal can vary with the direction of testing, not least in the through-thickness direction. Anistropy can be due to mechanical fibering-inclusions, porosity - but, this is not the concern of this paper. Plastic anisotropy - Plow stress, work-hardening behaviour - which results from crystallographic preferred or ientation, giving the metal a 'texture', can be varied in a sheet metal by altering the sequence and nature of the thermal and mechanical treatments which are used in its manufacture. In fact when it is realised that in rolling a coil from an ingot, the dispostion of the metal to the processing machinery is generally unchanged throughout the deformation and this cons is ts of reducing the metal thicliness by a factor of $~ 500$ it is not surprising that anisotropy is the rule rather than the exception.

Conventional tensile testine will not reveal plastic anisotropy as defined above. However, measurements of the changes in width strain and thickness strain durins uniaxial plastic deformation will indicate anisotropy, and their ratio;

$$
r=\frac{\epsilon_{W}}{\epsilon_{t}}
$$

is called the strain ratio, or commonly the $\underline{r}$ value. If this value is unity the material is isotropic and deviation is recognised as anisotropy.

Variation in $r$ with direction of testing in the sheet plane is termed planar anisctropy $(\Delta r)$ and nanifests itself as 'earing in a drawn axisymmetrical cup.

In metals with $r>I$ an increased resistance to yielding in biaxial tension is found and the converse for $r<1$ materials. Hosford and Backofen ${ }^{1}$ have coined the phrases 'texture hardening' and 'texture softening' to describe these phenomena. Clearly, the number of slip systems which can operate will affect the value of the strain ratio attainable. A material with an infinity of systems will be inevitably isotropic, while a metal with one slip direction, in one slip plane, and this, say, aligned parallel to the plane of the sheet will show $r=\infty$. Additionally, this metal would give $r=0$ for a specimen cut normal to the sheet surface. Face-centred cubic metals deform plastically on 12 equivalent systems, and in commercial polycrystalline sheet, $r_{\max }=1$ can be obtained ${ }^{2}$. Generally, the strain ratio value is around $0.6^{3}$. In body-centred cubic metals, four slip directions can 'choose' from a packet of slip planes, all of similar type. Although more slip systems can be written down for b.c.c. deformation, the modes are in fact more restrictive than in f.c.c. metals, and consequently higher and lover strain ratios can be obtained. Values from 0.3 to 2.2 have been measured for extra-deepdrawing steels ${ }^{4}$. In close-packed hexagonal metals a single slip direction is normally operative in one of three planes, and so, depending on the angle between the slip direction and the sheet plane, very high or very low strainratios will be observed. Values of 0.12 have be en reported in zinc ${ }^{5}$, while in zirconium values as high as 9.0 have been quoted ${ }^{6}$. 
Texture hardening is a useful, phenomenon. A recent study conducted by an aircraft company and reported by Babel and $\operatorname{Corn}^{7}$ showed that a $14.7 \%$ weight saving could be achieved by a redesign involving a change from an aluminium alloy to a titanium one. However, by taking into account the biaxial properties of titanium in addition, this saving could be increased to $21.0 \%$.

The purpose of the present work is to investigate the behaviour of a number of metals exhibiting a wide range of strain ratios in uniaxial and biaxial tension to test present anisotropic plasticity theory, and to show the advantages and disadvantages of this phenomenon in engineering applications.

The Mises criterion states:

$$
\left(\sigma_{1}-\sigma_{2}\right)^{2}+\left(\sigma_{2}-\sigma_{3}\right)^{2}+\left(\sigma_{3}-\sigma_{1}\right)^{2}=2 \sigma_{y}^{2}
$$

where $\sigma_{1}, \sigma_{2}$ and $\sigma_{3}$ are the stresses along orthogonal axes as shown in Figure la and $\sigma_{y}$ is the yield stress in uniaxial tension. For sheet loading $\left(\sigma_{3}=0\right)$ this reduces to:

$$
\sigma_{1}^{2}-\sigma_{1} \sigma_{z}+\sigma_{z}^{2}=\sigma_{y}^{2}
$$

Hill's yield criteria for anisotropic materials (again $\sigma_{3}=0$ ) can be written:

$$
\sigma_{1}^{2}+\sigma_{2}^{2}-\sigma_{1} \sigma_{2}\left(\frac{2 r}{r+1}\right)=\sigma_{y}^{2} \text {, where } r \text { is the strain ratio previous } 1 y
$$

discussed. This equation assumes planar isotropy, i.e. $\Delta r=0$ which is not true for many metals. The problem of a value for $r$ in a biaxial situation then must be solved, and at presert the most satisfactory solution is to define it as:

$$
\bar{r}=\frac{r_{0}+2 r_{45}+r_{90}}{4}
$$

or some variant on this theme depending on the number of directions in which tests are made. Figure lb shows plane-stress yield loci for various values of $r(\Delta r=0)$ and the texture hardening in the tension-tension quadrant and texture softening in the tension-compression quadrant should be noted.

The effect of $\underline{x}$ on yield and flow in uniaxial and balanced biaxial tension is the subject of the present investigation, and for a stress ratio $\left(\frac{\sigma_{2}}{\sigma_{1}}\right)$ of unity, it can be shown that:

$$
\frac{\sigma_{b}}{\sigma_{y}}=\sqrt{\frac{r+1}{2}}
$$

where $\sigma_{b}$ is the biaxial yield stress. 
It should be remembered that yield criteria are formulated for ideal elastic/plastic materials; the practical determination of $\sigma_{j}$ and $\sigma_{\mathrm{y}}$ especially the former - are virtually impossible; however, it is possible to take values of stress at low strains and measure the ratio of proof strengths at a specified strain.

With regard to the post-yield part of the stress-strain curves, Mellor and Bramley have suggested the prediction of the balanced biaxial curve from the uniaxial data using the following expressions:

$$
\begin{aligned}
& \sigma_{3}=\sqrt{\frac{1+\vec{r}}{2}} \cdot \sigma_{\mathrm{av}} \\
& \epsilon_{3}=\sqrt{\frac{2}{1+\underline{\underline{r}}}} \cdot \epsilon_{\mathrm{av}}
\end{aligned}
$$

$\sigma_{a v}$ is the average true stress at strain $\epsilon_{a v}$, the average true strain, the averages be ing determined from the uniaxial data and 'weighted' according to an analogous formula to that for $\overline{\underline{r}}$.

\section{Experimental}

The uniaxial tensile tests were carried out on an Instron machine at a crosshead speed of $0.2 \mathrm{in} / \mathrm{min}$. Specimens were machined according to BSI8, and a load-extension curve was dram, using a $2^{\prime \prime}$ gauge-length strain-gauge extensometer on the specimen up to fracture. $\sigma$ was calculated from the relationship:

$$
\sigma=\frac{P}{A_{0}}(I+e)
$$

where $P$ is the instantaneous load, $A_{0}$ the original cross-sectional area and $e$ the engineering strain, and $\epsilon$ from:-

$$
\epsilon=\ln (I+e)
$$

The biaxial curves were determined using a Mand Precision Engineering Co. Itd., test extensometer, developed by Johnson and Duncan ${ }^{9}$ where 0 is computed from:

$$
\sigma=\frac{P \rho}{2 t}
$$

where $\rho$ is the radius of curvature and the current thickness, and $\epsilon$ from:

$$
\epsilon=\ln \left(\frac{a}{d_{c}}\right)
$$

where $d_{0}$ and $d_{I}$ are the initial and current diameters of a reference circle. 
Results

The results are shown in Figures $2,3,4,5,6,7,3,69$ and 10 . It vill be seen (Fisure 2 ) that the relationship between $\frac{b}{\sigma_{y}}$ and $r$ (at a strain of 0.01 ) is similar in shape to the predicted one, but displaced to a higher stress level. The exceptions here are the two samples of commercial-purity aluminjum tested, one sopt and one hard-rolled.

In Figures 3 through 10 the experimental and predicted curves are drawn. For materials with $r>I$ the predicted curves are quite good approximations to the determined ones, especially titanium and zirconium. For $r<I$ the discrepancies are very marked. In no cases are the biaxial curves below the uniaxial, as predicted, but in some cases the biaxial curves start lower and then cross. In the case of commercial-purity zinc, the uniaxial curves themselves are so widely spaced that the use of an averaging procedure is a doubtful operation.

\section{Conclusions}

Present anisotropic plasticity theory falls down for materials exhibiting $r<I$ ard even for materials of $r \approx i$; the superposition of uniaxial and biaxial curves is not good. In general, the flow curve in biaxial tension falls above the uniaxial one regardless of the state of anisotropy. However, at the low - strain end of the curves there is a tendency for the curves to cross, especially the curves for the low $-\bar{r}$ materials. Explanations for this must be microstructural rather than phenomenological and the following tentative qualitative picture is offered. It is permissible to equate belanced biaxial tension with through-thickness compression plus a hydrostatic tension wich does not affect yielding. In a low $\overrightarrow{\vec{r}}$ metal the operative slipsystems $w i l l$ tend to be aligned to cause strain in the thiclmess direction and so under this stress sytem slip will occur readily on many planes, many dislocations will move and entangle and a high initial rate of work-hardening will result.

It is not possible to allow for this sort of behaviour in plasticity theory. In the case of zinc, it will be observed that the $\sigma$, $\epsilon$, curve falls at high strains. This is due to the formation of voids during straining ( $F i g$. II) and the consequent violation of the volume-constancy axiom.

Again, during deformation - particularly of face-centred cubic metals, grain rotation occurs, and $\underline{r}$ may alter during deformation. This is another uncharted variable.

A limitation of these experiments is the inability to measure biaxial stressustrain at very low strains, due to the uncertainty of radius of curvature measurements at low bulging pressures.

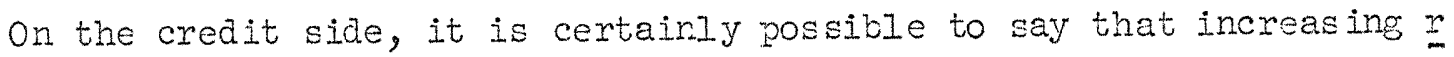


will raise the baxial flow curve, and in practical engineering applications it is in this direction that the benefit lies; approximate calculations of $\sigma_{b}$ from $\sigma_{y}$ and $\bar{x}$ would be in order. An additional benefit is that $\sigma_{b} i s$, in general, most likely to be greater than $\sigma_{\mathrm{y}}$ for all metals, but here $\sigma_{b}$ must be masured experimentally. However, the bulge test procedure used in this work is not tedirus and tends to be quite reproducible.

Sone of those interested in sheet metal formability and particularly the formability of sheet steel regard $n$, the exponent in the expression $\sigma=K \epsilon^{n}$, as a useful parameter. This can be obtained quite easily from the tiaxial curves (those which conform to this relationship) and in general appears to be of similar value to that obtained in uriaxial tension. Maximum strains obtained in this work accord approximately with Hill's prediction for a circular diaphragm and are given in Table $I$. The good agreement of the mild steels in particular should be noted.

More work is needed particularly with metals of $r \leqslant 1$. Bott and Pearce ${ }^{3}$ showed that, with commercial-purity aluminium, quite large changes in strain could be affected by cold rolling, without sienificant changes in $\overline{\underline{r}}$. It is with material of this sort that further work will be carried out.

Acknowledgements

I would Iike to thank Mr. I.C. Drinkwater for his careful work on the stress-strain curves and Dr. P.B. Mellor for a helpful discussion. 
TABIE I

Predicted and measured effective strains at instability in a circular diaphragm.

Materials (all in the annealed conditions).

$70 / 30$ Brass

Cumcontaining steel

Tiacontaining steel

Rimming steel

Commercial-purity aluminium

Commercial-purity zinc

$$
n=\bar{E}_{u}
$$

0.43

0.24

0.18

0.26

0.35

0.36

$$
\bar{\epsilon}_{\text {meas biaxial }} \begin{gathered}
\text { balanced } \\
\text { tension }
\end{gathered}
$$$$
\tilde{E}=\frac{4}{11}(2 n+I)
$$

0.73

0.68

0.59

0.54

0.50

0.49

0.51

0.55

0.54

0.62

0.57

0.63 
References

1. Hosford, W.F. Jnr., and Baclofen, W.A.

2. Pearce, Roger and Joshi, P.G.

3. Pearce, Roger and Bott, C.H.

4. Heyer, R. and Newby, J.

5. Ackinson, $M$.

6. Jurson, F.R.

7. BabeI, H.W. and Corn, D.I.

8. Menor, P.B. and Brem].ey, A.IT.

9. Johrson, W. and Duncan, J.I.
Strength and plasticity in textured metals. Proceedings of the ninth Sagamore ordnance materials research conference, Syracuse University Press, 1965.

Relationship between the behaviour of a range of aluminium/magnesium alloys in uniaxial and biaxial tension.

A.S.M. Trans. Quart. 1964, 57, 399.

The effect of varying strain ratio on the hyaraulic bulging behaviour of aluminium sheet.

J. Aust. Inst. Metals. 1967, 12, 119.

Effect of mechanical properties on biaxial stretchability of low carb on steels. Paper to be presented at the $1968 \mathrm{National}$ S.A.E. Congress, Detroit. (Jamary, 1968).

Assessing nomal anisotropic plasticity of sheet metals.

Sheet Meta1 Industries, 1967, 44, 167.

Anisotropy of titanium sheet in unaxial tension.

A.S.M. Trans Quart. 1962, 55, 264.

A comparison of methods for correlative texturing with the biaxial strengths of titanium alloys.

Metals Eng. Quart. 1967, 7, 45.

Contribution to a discussion on 'Mechanical anisotropy in sheet metals". March, 1965, London.

The use of the biaxial test extensometer. Sheet Metal Industries, 1965, 42, 281. 


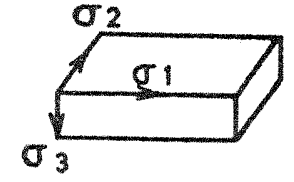

FIGURE ia.

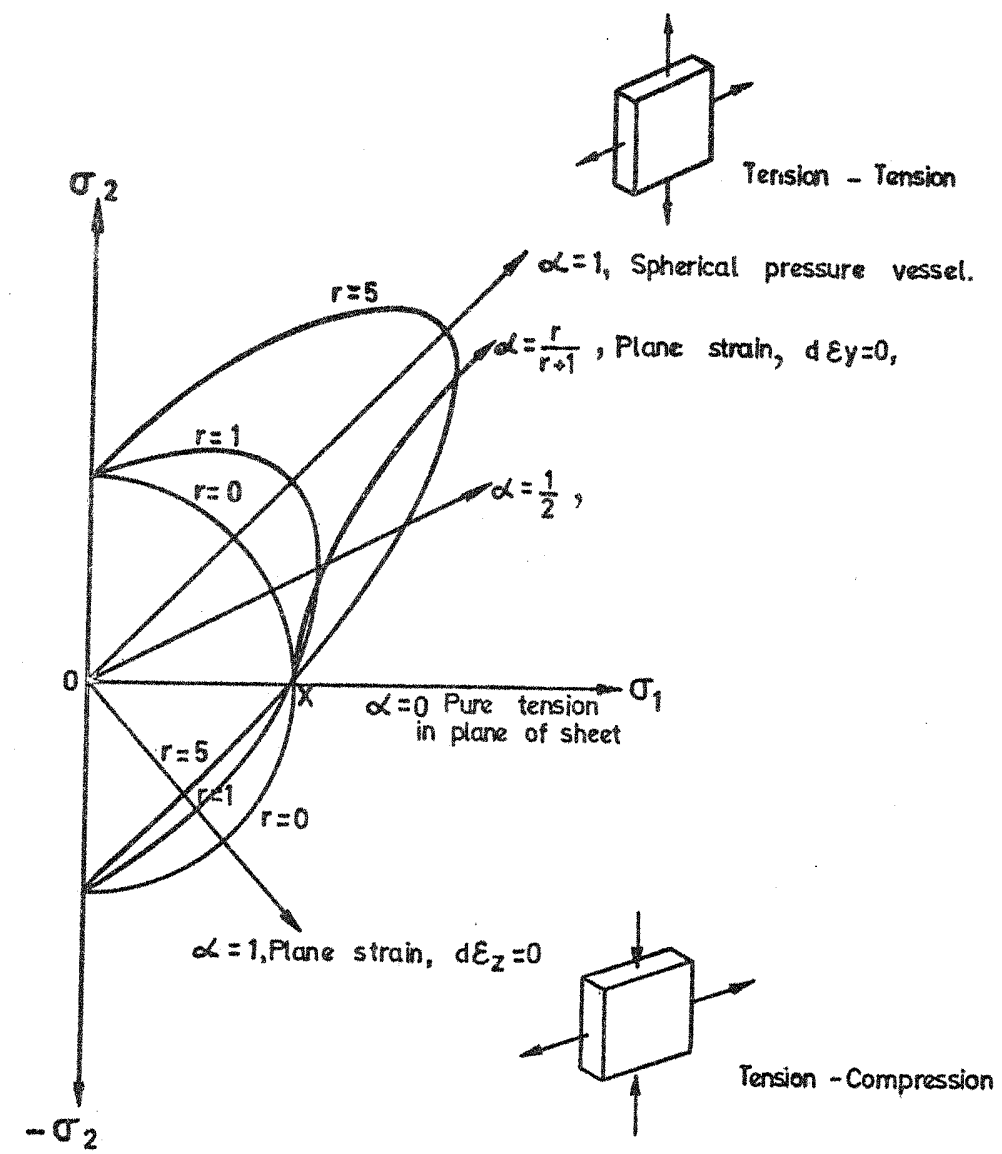

FIGURE 1b. Plane stress yield loci. 


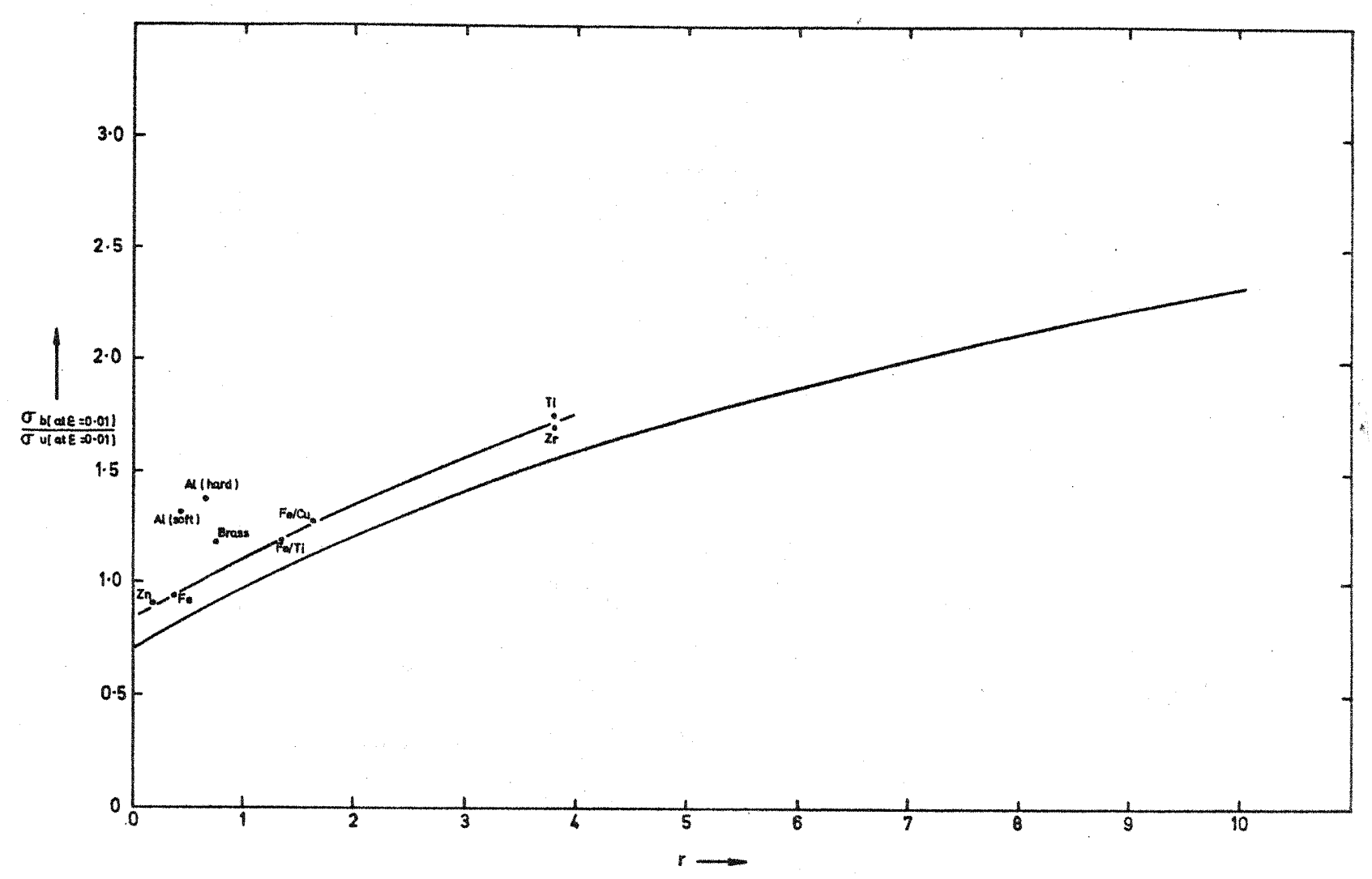

FIG. 2 


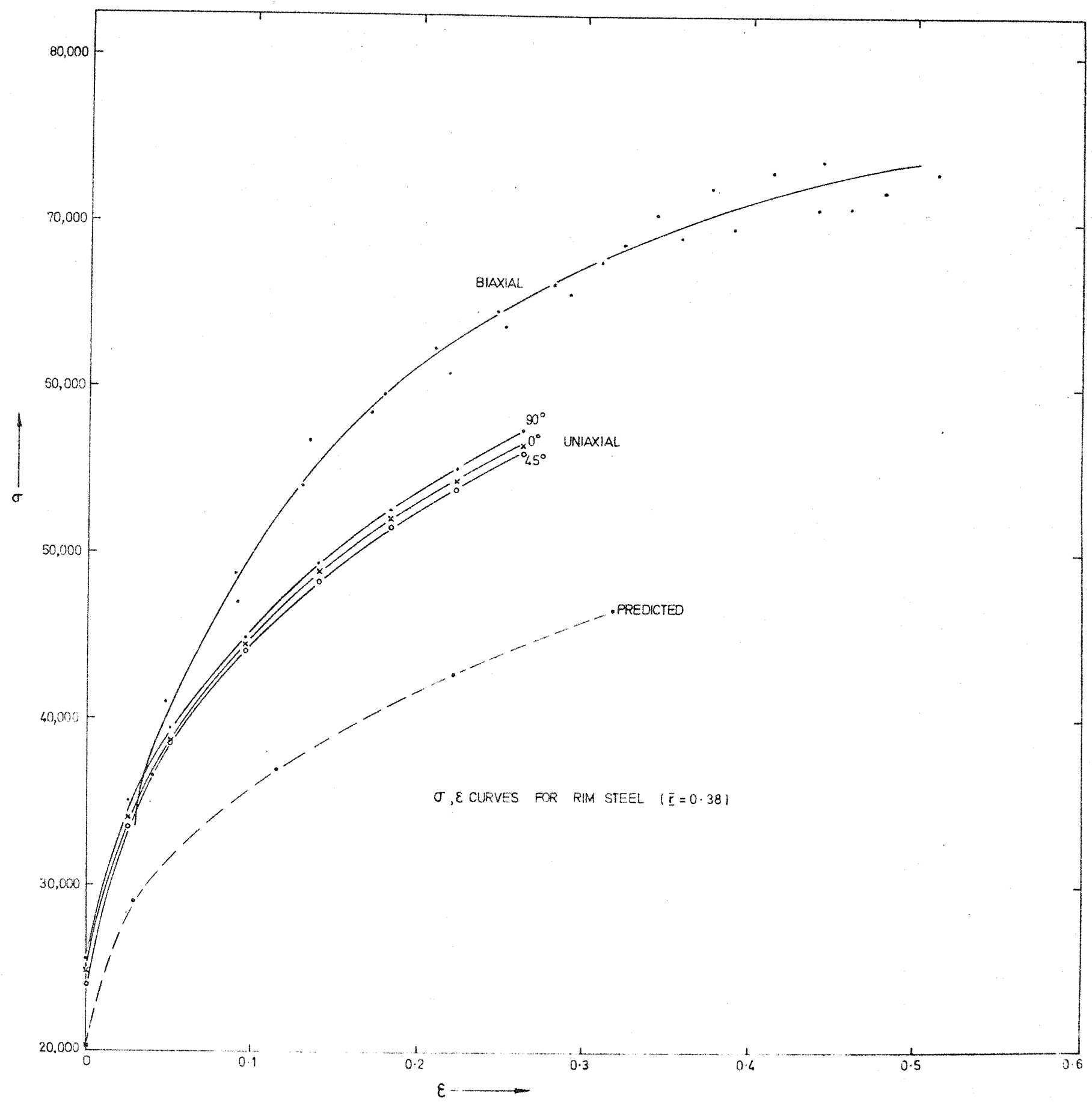

FIG. 3 


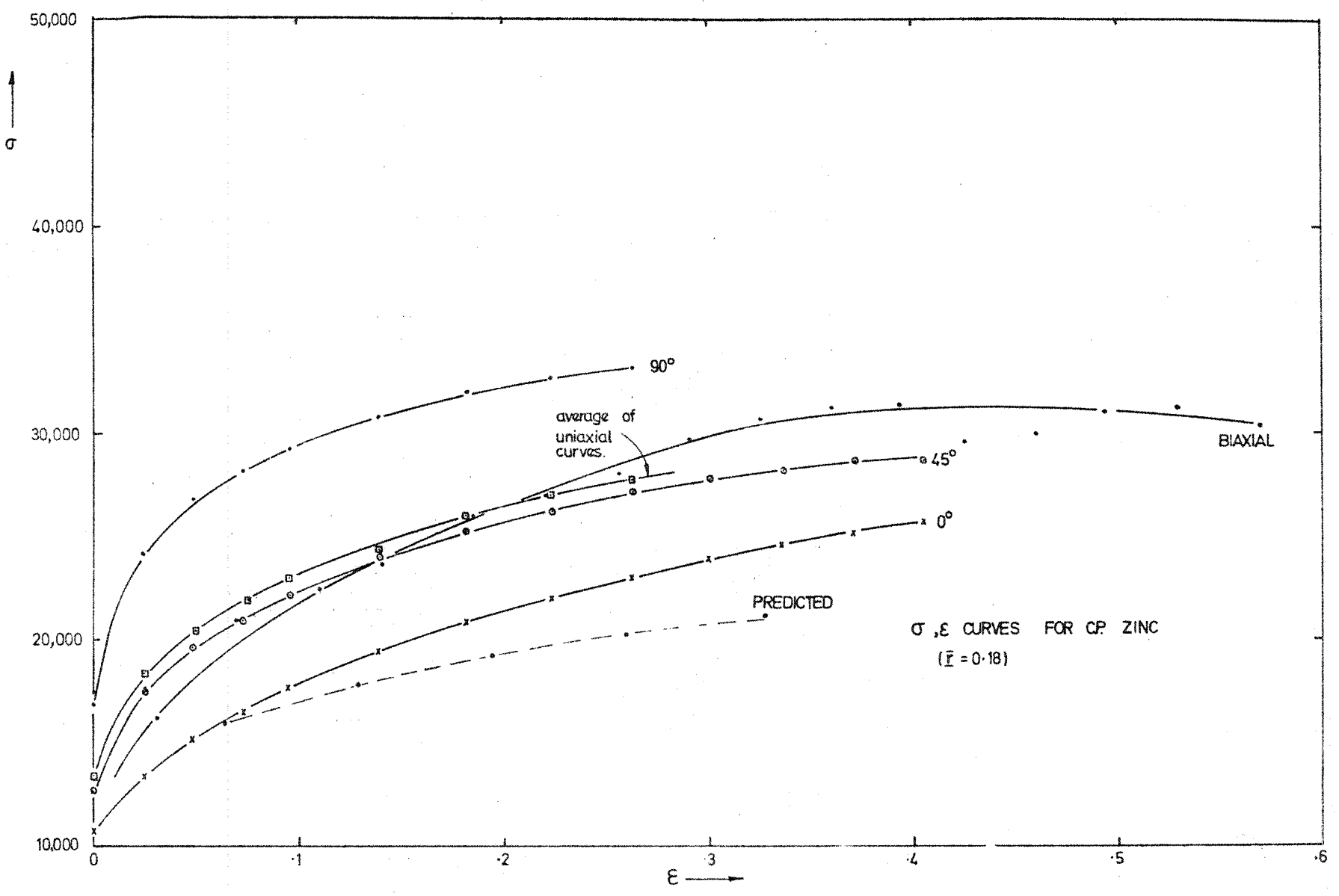

FIG. 4

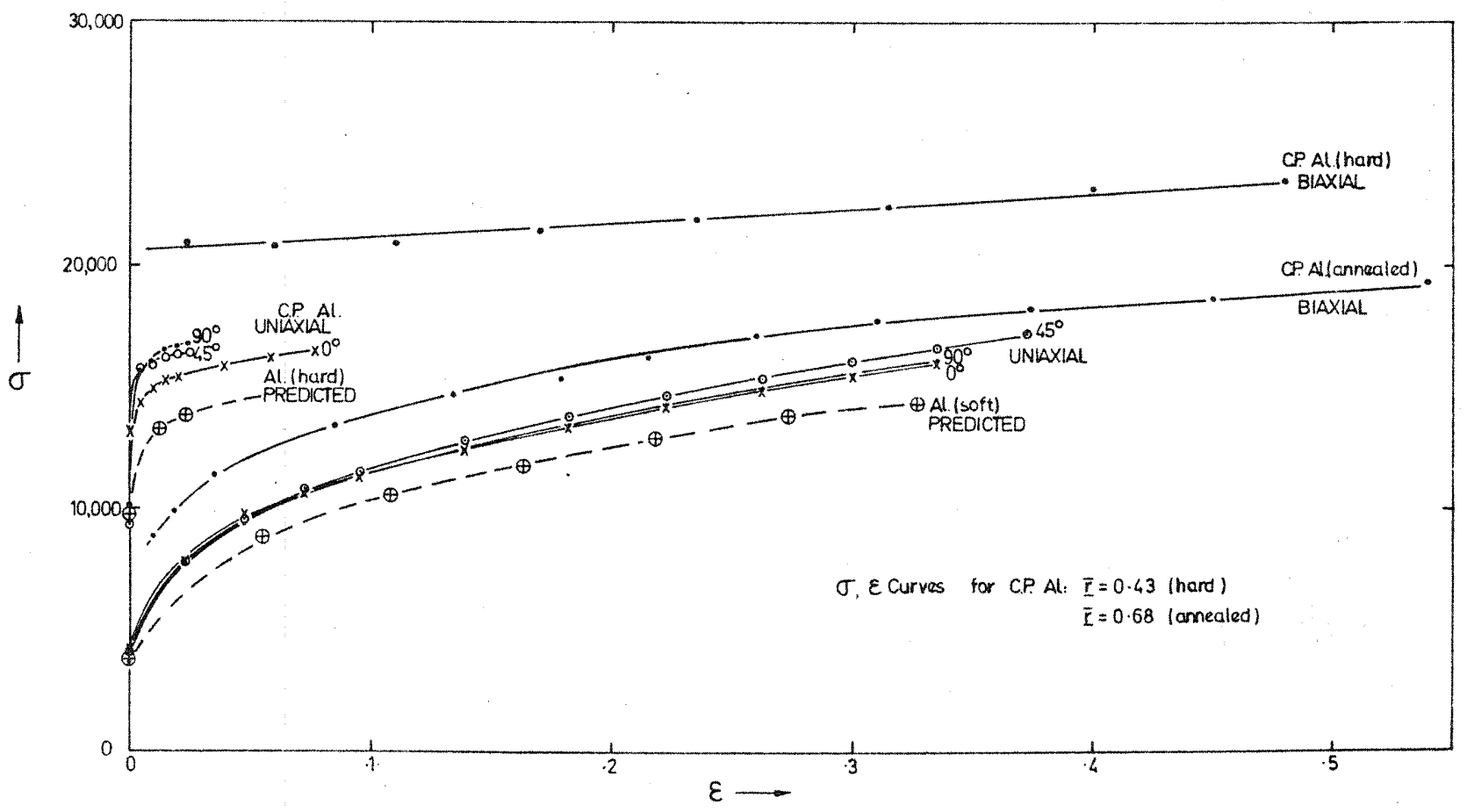

FIG. 5 


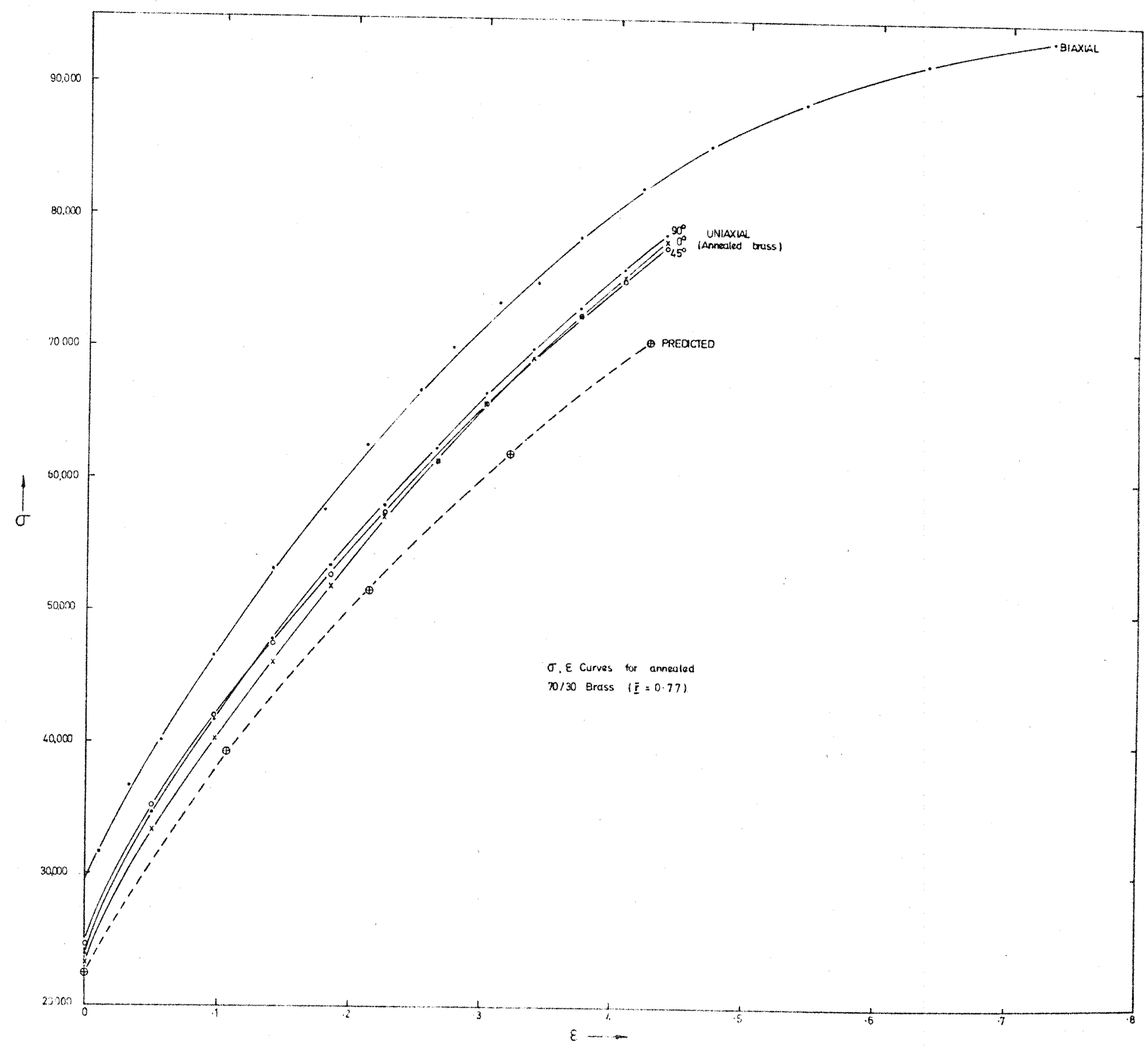

FIG. 6 


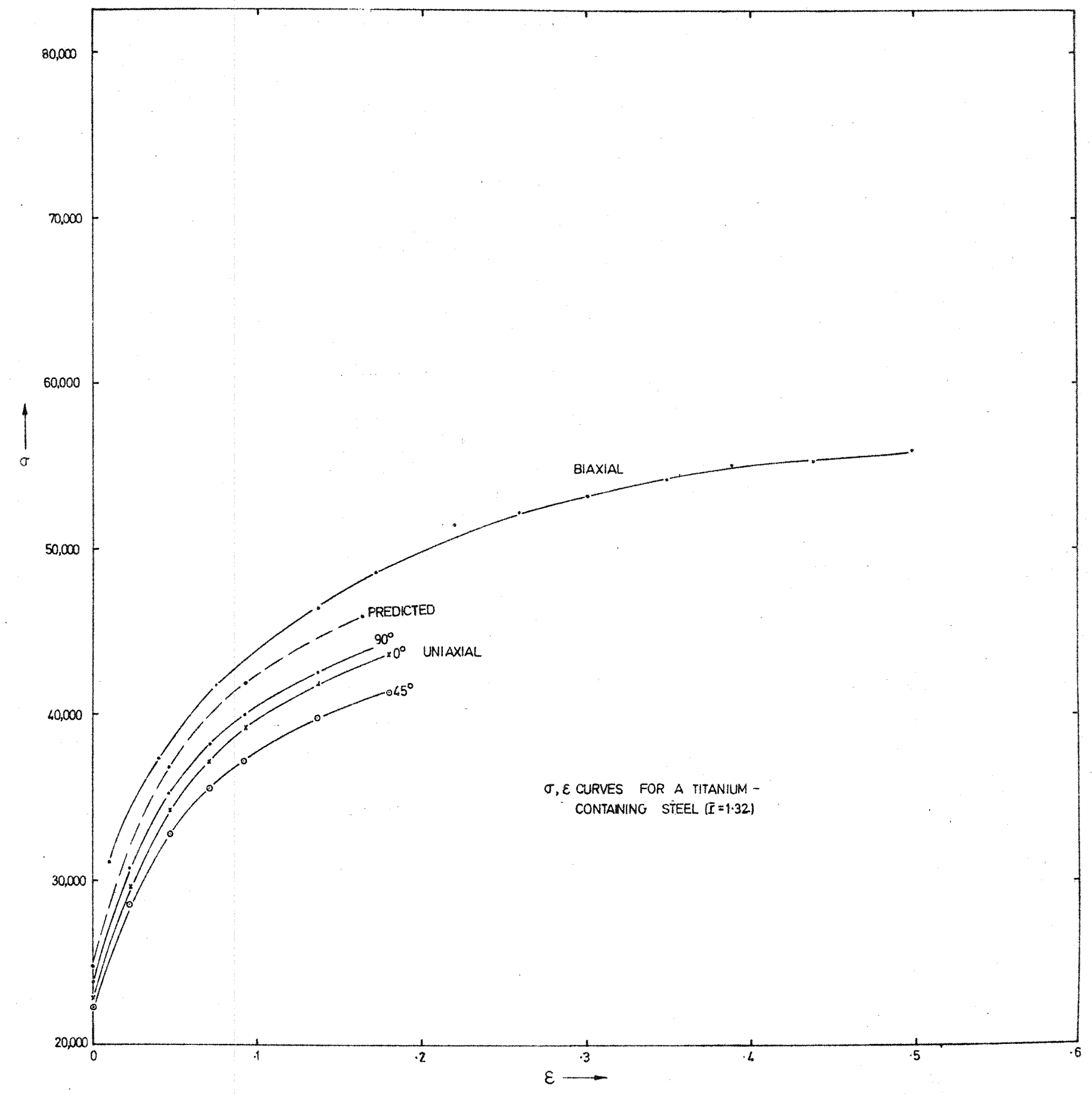

FIG. 7 


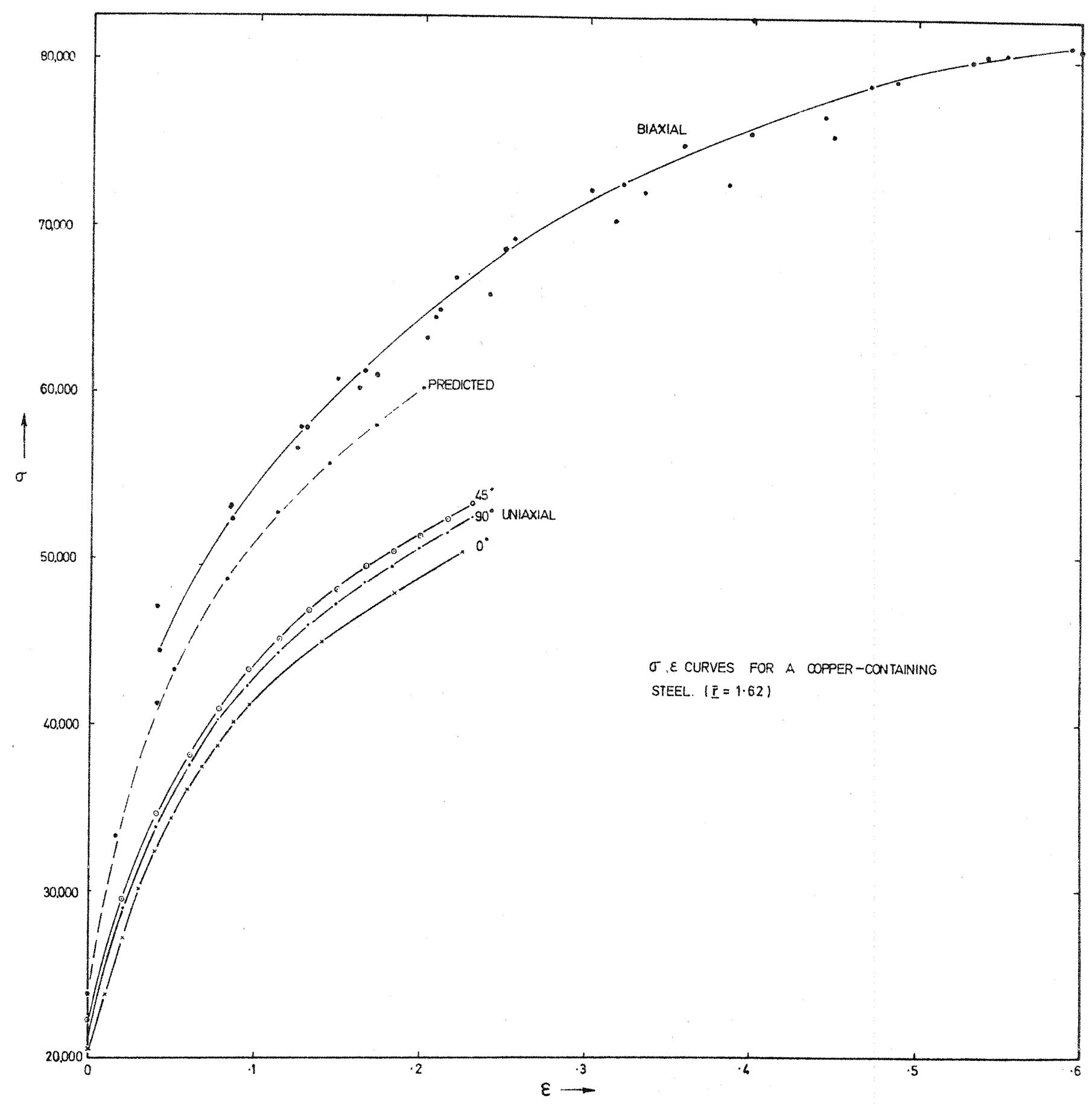

FIG. 8 


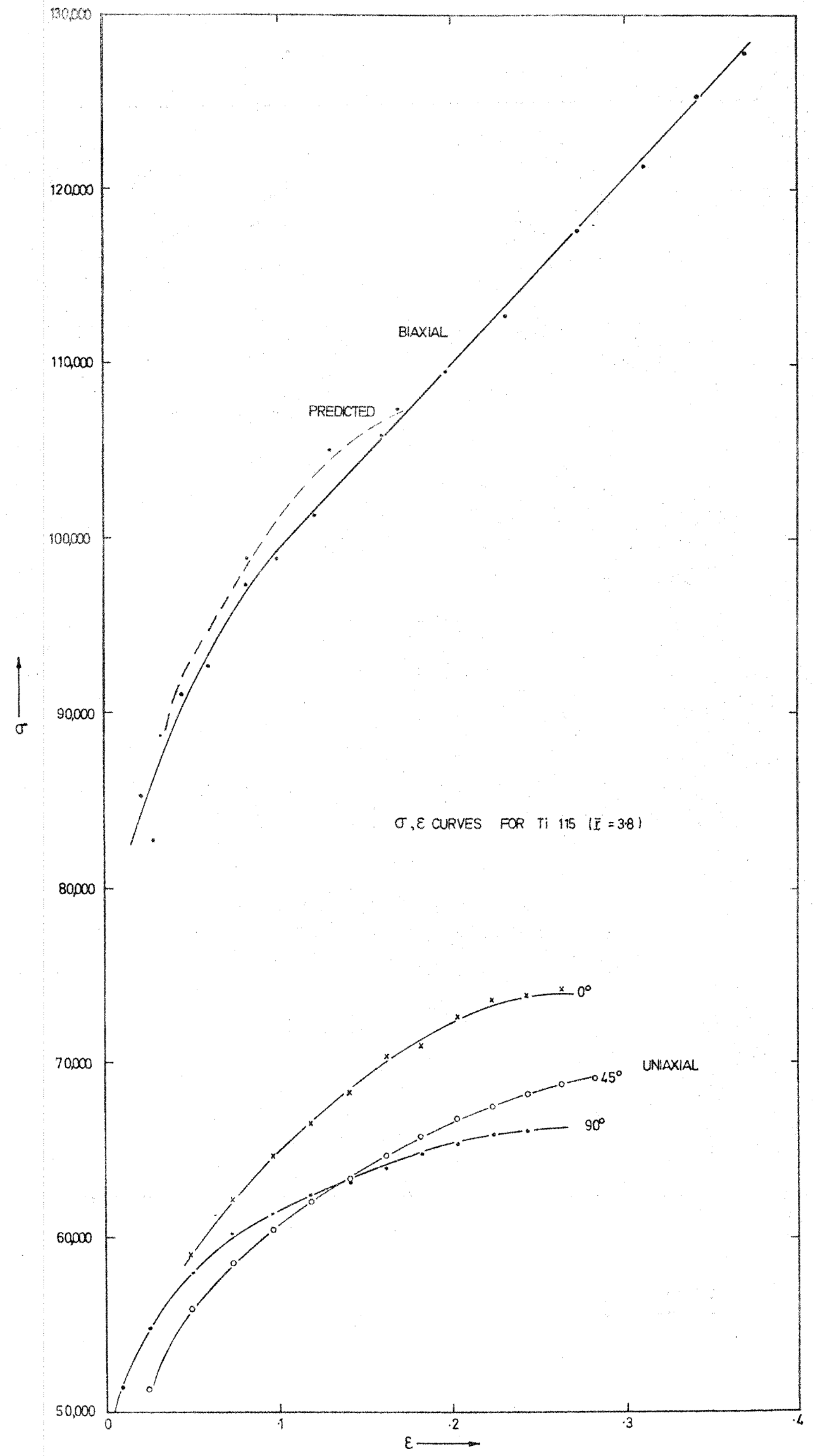

FIG. 9 


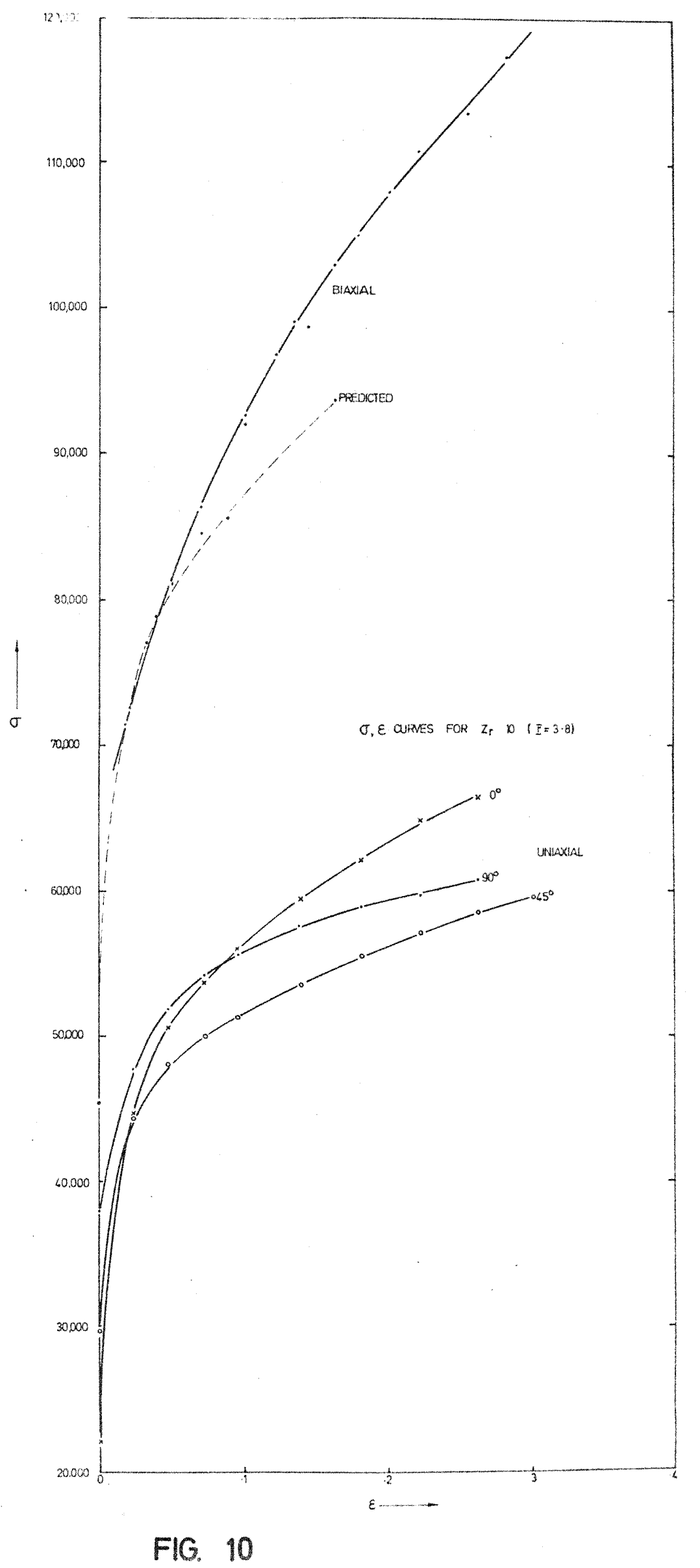




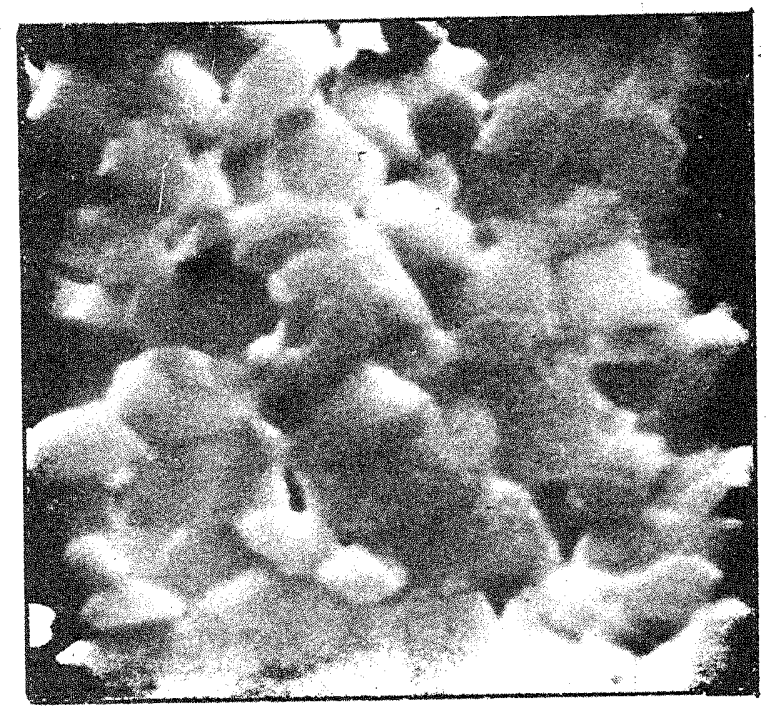

FIG. 11. 